\title{
Hausdorff Discretizations of Algebraic Sets and Diophantine Sets
}

\author{
Mohamed Tajine and Christian Ronse \\ Laboratoire des Sciences de l'Image, de l'Informatique et de la Télédétection \\ (LSIIT, UPRES-A CNRS 7005) \\ Boulevard Sébastien Brant, F-67400 Illkirch, France \\ \{tajine,ronse\}@dpt-info.u-strasbg.fr
}

\begin{abstract}
This paper is a continuation of our works $[12,13,15,16,17,18]$ $[21,22]$ in which we study the properties of a new framework for discretization of closed sets based on Hausdorff metric. Let $F$ be a nonempty closed subset of $\mathbb{R}^{n} ; S \subseteq \mathbb{Z}^{n}$ is a Hausdorff discretization of $F$ if it minimizes the Hausdorff distance to $F$.

We study the properties of Hausdorff discretizations of algebraic sets. Actually we give some decidable and undecidable properties concerning Hausdorff discretizations of algebraic sets and we prove that some Hausdorff discretizations of algebraic sets are diophantine sets. We refine the last results for algebraic curves and more precisely for straight lines.
\end{abstract}

Keywords: Algebraic set, diophantine set, Hausdorff discretization, homogeneous metric.

\section{Introduction}

We have introduced a new framework for discretization of closed sets based on the Hausdorff metric : Hausdorff discretization. The basic idea is to select as possible discretizations of a Euclidean set $F$ all discrete sets $S$ such that the Hausdorff distance between $F$ and $S$ is minimal. This leads to several possible choices for such a discretization.

Our framework is as follows: given a metric $d$ on $\mathbb{R}^{n}$, let $F$ be a non-empty closed subset of $\mathbb{R}^{n}$; then $S \subseteq \mathbb{Z}^{n}$ is a Hausdorff discretization of $F$ if it minimizes the Hausdorff distance $H_{d}(S, F)$ to $F$. Some geometrical and topological properties of Hausdorff discretization and its comparison with other discretization schemes are studied in the serie of papers [12,13,15,16,17,18,21,22].

In this paper, we study the Hausdorff discretizations of particular sets : real algebraic sets. After recaling the fundametal properties of Hausdorff discretizations and the refinement of their properties for the class of homogeneous metrics, we give some decidable and undecidable properties concerning Hausdorff discretizations of algebraic sets and we prove that some Hausdorff discretizations of algebraic sets are diophantine sets. Some of these results are proven by using the Tarski's elimination algorithm concerning the decidability of first order logic for elementary algebra $[9,14,19,20]$. The time complexity of Tarski's elimination 
algorithm, is in the general case, very high [5]. We refine also the last results for algebraic curves and more precisely for straight lines. Actually we prove that the Hausdorff discretizations of straight lines can be discribed by using two periodic diophantine sets. This last result generalize the results of [1] concerning the supercover discretization of straight lines.

In this paper, for simplifying the notation in the notions which depend on a metric $d$, we do not refer explicitely to $d$, except when there is an ambiguity.

This paper is divided into eight sections. In the second section we briefly recall classical notions of metric space and Hausdorff space. In the third section, we introduce the Hausdorff discretizations. Section 4 deals with homogeneous metrics, while Section 5 introduc real algebraic sets, real semi-algebraic sets and diophantine sets. In the sixth section we characterize some Hausdorff discretizations of real algebraic sets. In the seventh section, we refine the last results for algebraic curves and more precisely for straight lines. The last section is a conclusion.

The proofs are not given here, the proofs the the results of sections 2,3 and 4 can be found in $[12,15]$.

\section{Some Metric Notions and Hausdorff Metric}

We assume that the reader is familiar with classical notions of topological space, metric space and normed space, see for example $[3,6,7]$. We introduce here our notations, most of them are recalled in [12,15,21,22].

Definition 1. Let $(\mathcal{E}, d)$ be a metric space, and let $p \in \mathcal{E}$ and $r \in \mathbb{R}^{+}$,

$$
\mathcal{B}_{r}^{d}(p)=\{x \in \mathcal{E} \mid d(x, p) \leq r\} .
$$

$\mathcal{B}_{r}^{d}(p)$ is called the ball of center $p$ and of radius $r$.

Let $E \subseteq \mathcal{E}$. int $(E)=\left\{x \in E \mid \exists r>0, \mathcal{B}_{r}^{d}(x) \subseteq E\right\}$, int $(E)$ is called the interior of $E$ (i.e. $\operatorname{int}(E)$ is the largest open set contained in $E$ ).

In all the following, all topological notions in a metric space $(\mathcal{E}, d)$ are considered relatively to the topology induced by $d$. All metrics used in this paper are induced by norm. So if $N$ is a norm over $\mathcal{E}$, then the function $d_{N}$ such that: $\forall x, y \in$ $\mathcal{E}, d_{N}(x, y)=N(x-y)$ is called the metric over $\mathcal{E}$ induced by $N$.

\section{Example:}

Let $x=\left(x_{1}, x_{2}, \ldots, x_{n}\right) \in \mathbb{R}^{n}$. Then $\forall p \geq 1,|x|_{p}=\sqrt[p]{\left|x_{1}\right|^{p}+\ldots+\left|x_{n}\right|^{p}}$ and $|x|_{\infty}=\max _{1 \leq i \leq n}\left|x_{i}\right|$ are a norms over $\mathcal{E}=\mathbb{R}^{n}$. The metrics induced by these norms are denoted $d_{p}$ and $d_{\infty}$ respectively. 


\subsection{Hausdorff Metric}

The definitions and results presented in this subsection can be found in [3,6].

Definition 2. Let $(\mathcal{E}, d)$ be a metric space; $\mathcal{H}(\mathcal{E})$ is the set of non-empty compact subsets of $\mathcal{E}, \mathcal{F}(\mathcal{E})$ the set of closed subsets of $\mathcal{E}$, and $\mathcal{F}^{\prime}(\mathcal{E})$ the set of non-empty closed subsets of $\mathcal{E}\left(\right.$ i.e. $\left.\mathcal{F}^{\prime}(\mathcal{E})=\mathcal{F}(\mathcal{E}) \backslash\{\emptyset\}\right)$.

On $\mathcal{H}(\mathcal{E})$, we will define a metric $H_{d}$, such that if $(\mathcal{E}, d)$ is a complete metric space then $\left(\mathcal{H}(\mathcal{E}), H_{d}\right)$ is a complete metric space.

Definition 3. Let $(\mathcal{E}, d)$ be a metric space and let $A, B \in \mathcal{H}(\mathcal{E})$. We define the oriented Hausdorff metric from $A$ to $B$ by $h_{d}(A, B)=\max _{a \in A}(d(a, B))$ where $d(a, B)=\min _{b \in B}(d(a, b))$.

Definition 4. Let $(\mathcal{E}, d)$ be a metric space. The Hausdorff distance between two compact sets $A, B \in \mathcal{H}(\mathcal{E})$ is defined by $H_{d}(A, B)=\max \left(h_{d}(A, B), h_{d}(B, A)\right)$.

\section{Remark:}

Let $\mathcal{F}^{\prime}(\mathcal{E})$ be the set of non empty closed set of $\mathcal{E}$. Then, the functions $h_{d}$ and $H_{d}$ can be extended in natural way as function from $\mathcal{F}^{\prime}(\mathcal{E}) \times \mathcal{F}^{\prime}(\mathcal{E})$ to $\mathbb{R}^{+} \cup\{+\infty\}$ (note: in Definition 3, the "max" (resp. "min") becomes a "sup" (resp. "inf"))

$H_{d}$ is a "generalized metric" on $\mathcal{F}^{\prime}(\mathcal{E})$ in the sense that it satisfies the axioms of metric, but can take infinite values.

\section{Hausdorff Discretization}

In this section, we recal our framework of discretization based on Hausdorff metric. Our results are proved in [15]. In the rest of this paper we assume that we have as metric space $\left(\mathbb{R}^{n}, d\right)$, where $d$ is a metric induced by a norm on $\mathbb{R}^{n}$, and as a discrete space $\mathcal{D}_{\rho}=\rho \mathbb{Z}^{n}$, for $\rho>0$. For a such distance, $M \subseteq \mathcal{D}_{\rho}$ implies that $M \in \mathcal{F}\left(\mathbb{R}^{n}\right)$.

In all the following, we assume that $\rho>0$.

Definition 5. Let $d$ be a metric on $\mathbb{R}^{n}$ and $\rho>0$. The covering radius of the metric $d$ in $\mathcal{D}_{\rho}$ is

$$
r_{c}(\rho)=\sup _{x \in \mathbb{R}^{n}}\left(d\left(x, \mathcal{D}_{\rho}\right)\right)
$$

Notation:

Let $d$ be a metric on $\mathbb{R}^{n}$ and $r \geq 0$;

$$
\forall F \in \mathcal{F}^{\prime}\left(\mathbb{R}^{n}\right), \Delta_{r}^{d}(F, \rho)=\left\{p \in \mathcal{D}_{\rho} \mid \mathcal{B}_{r}^{d}(p) \cap F \neq \emptyset\right\} .
$$

Let $F$ be a non-empty closed subset of $\mathbb{R}^{n} ; M \subseteq \mathcal{D}_{\rho}$ is a Hausdorff discretization of $F$ in $\mathcal{D}_{\rho}$ if it minimizes the Hausdorff distance to $F$. In this section, we study the properties of Hausdorff discretizations. In [12,13,21,22], we have studied the Hausdorff discretizations when $F$ is a compact set. 
Definition 6. Let $F \in \mathcal{F}^{\prime}\left(\mathbb{R}^{n}\right)$.

- A set $M \subseteq \mathcal{D}_{\rho}$ is a Hausdorff discretization of $F$ in $\mathcal{D}_{\rho}$ if $H_{d}(F, M)=\inf \left(\left\{H_{d}(F, S) \mid S \subseteq \mathcal{D}_{\rho}\right\}\right)$.

- $\mathcal{M}_{H}(F, \rho)=\left\{M \subseteq \mathcal{D}_{\rho} \mid H_{d}(F, M)=i n f_{S \subseteq \mathcal{D}_{\rho}}\left(H_{d}(F, S)\right)\right\}$ is the set of Hausdorff discretizations of $F$.

- $\Delta_{H}(F, \rho)=\left(\bigcup_{M \in \mathcal{M}_{H}(F, \rho)} M\right)$ is called the maximal Hausdorff discretization of $F$ in $\mathcal{D}_{\rho}$ (i.e. The justification of this definition is given in the following Theorem $)$.

- The value $r_{H}(F, \rho)=\sup _{x \in F}\left(d\left(x, \mathcal{D}_{\rho}\right)\right)$ is called the Hausdorff radius of $F$ in $\mathcal{D}_{\rho}$ for the metric $d$.

We characterize now the Hausdorff discretizations.

Theorem 1. Let $F \in \mathcal{F}^{\prime}\left(\mathbb{R}^{n}\right)$; then:

- $\mathcal{M}_{H}(F, \rho)$ is nonvoid and for $M \in \mathcal{M}_{H}(F, \rho), H_{d}(F, M)=r_{H}(F, \rho)$;

- for a family $\left(M_{i}\right)_{i \in I}$ of members of $\mathcal{M}_{H}(F, \rho), \bigcup_{i \in I} M_{i} \in \mathcal{M}_{H}(F, \rho)$ and so $\Delta_{H}(F, \rho) \in \mathcal{M}_{H}(F, \rho)$;

- if $\left(M_{n}\right)_{n \in N}$ is a decreasing sequence in $\mathcal{M}_{H}(F, \rho)$ (relatively to the set inclusion) then $\bigcap_{n \in N} M_{n} \neq \emptyset$ and $\bigcap_{n \in N} M_{n} \in \mathcal{M}_{H}(F, \rho)$;

- $\Delta_{H}(F, \rho)=\left\{p \in \mathcal{D}_{\rho} \mid d(p, F) \leq r_{H}(F, \rho)\right\}$;

- $\mathcal{M}_{H}(F, \rho)$ is the set of all $M \in \mathcal{D}_{\rho}$ such that $M \subseteq \Delta_{H}(F, \rho)$ and $F$ is included in the union of balls of radius $r_{H}(F, \rho)$ centered about points of $M$;

- $r_{H}(F, \rho) \leq r_{c}(\rho)$;

- $\Delta_{H}(F, \rho)=\Delta_{r_{H}(F, \rho)}^{d}(F, \rho)$.

Definition 7. Let $p \in \mathcal{D}_{\rho}$, we define the the cell of center $p$ as

$$
\mathcal{C}(p, \rho)=\left\{x \in \mathbb{R}^{n} \mid d_{\infty}(x, p) \leq \frac{\rho}{2}\right\}
$$

Note that cells are closed and overlap only at their boundaries.

In Figure 1 we illustrate the construction of Hausdorff discretizations for a closed set $F$ : computing the Hausdorff radius (maximal distance from points of $F$ to the discrete space), one takes for $\Delta_{H}(F, \rho)$ all discrete points $p$ such that the ball of center $p$ and Hausdorff radius intersects $F$; any subset $M$ of $\Delta_{H}(F, \rho)$ such that the corresponding balls for $p \in M$ cover $F$, will be a Hausdorff discretization.

Definition 8. Let $d$ be a metric on $\mathbb{R}^{n}$ and $F \in \mathcal{F}^{\prime}\left(\mathbb{R}^{n}\right)$, the skeleton of $\Delta_{H}(F, \rho)$ is the set

$$
\mathcal{S} k(F, \rho)=\bigcap_{M \in \mathcal{M}_{H}(F, \rho)} M .
$$

Note that $\mathcal{S} k(F, \rho)$ is not generally a Hausdorff discretization of $F$. 

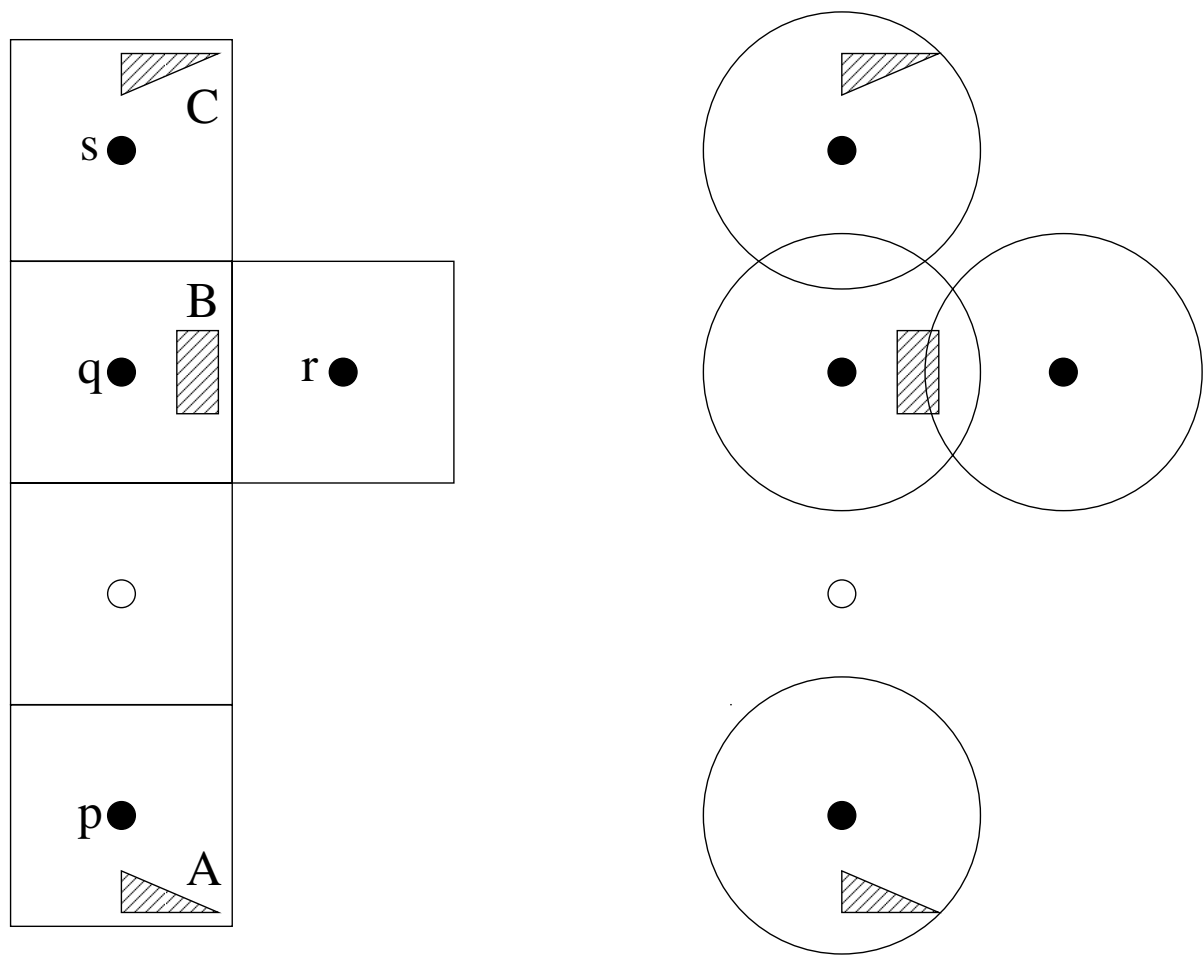

Fig. 1. Left: The set $F=A \cup B \cup C$ overlayed with discrete points $p, q, r, s$ and their square cells $C(p, 1), C(q, 1), C(r, 1), C(s, 1)$. Right: For $d=d_{2}$ (the Euclidean distance), the maximal Hausdorff discretization of $F$ is $\{p, q, r, s\}$; indeed, we show the circles of radius $r_{H}(F, 1)$ centered about these points. The unique other Hausdorff discretizing set of $F$ is $\{p, q, s\}$

\section{Homogeneous Metric and Hausdorff Discretization}

We present some properties of a homogeneous metric and we refine the characterization of Hausdorff discretizations for such metrics. Actually, we study the relationship between Hausdorff discretizations and covering discretizations. Again, results of this section are proved in [15].

Definition 9. A norm $N$ on $\mathbb{R}^{n}$ is homogeneous if for every $\left(x_{1}, \ldots, x_{n}\right) \in$ $\mathbb{R}^{n}$, every $\left(\varepsilon_{1}, \ldots, \varepsilon_{n}\right) \in\{-1,1\}^{n}$, and for every permutation $\sigma$ of $\{1, \ldots, n\}$, we have $N\left(\varepsilon_{1} x_{\sigma(1)}, \ldots, \varepsilon_{n} x_{\sigma(n)}\right)=N\left(x_{1}, \ldots, x_{n}\right)$. A metric induced by a homogeneous norm is called a homogeneous metric.

Theorem 2. Let $d$ be a homogeneous metric induced by a norm $N$, then:

- $r_{c}(1)=\frac{1}{2} N(1, \ldots, 1)$ and

- $\mathcal{B}_{\frac{1}{2}}^{d_{\infty}}(O) \subseteq \mathcal{B}_{r_{c}(\rho)}^{d}(O) \subseteq \mathcal{B}_{\frac{n}{2}}^{d_{1}}(O)$, for all $O \in \mathbb{R}^{n}$. 


\section{Examples:}

In $\mathbb{R}^{n}, \forall p \geq 1, d_{p}$ is a homogeneous metric and thus, $r_{c}(1)=\left(\frac{n^{\frac{1}{p}}}{2}\right)$, and $d_{\infty}$ is also a homogeneous metric and thus, $r_{c}(1)=\frac{1}{2}$.

Definition 10. Let $E \subseteq \mathbb{R}^{n}$, a subset $S \subseteq \mathcal{D}_{\rho}$ is called a covering discretization of $E$ in $\mathcal{D}_{\rho}$, if $\forall p \in S, E \cap \mathcal{C}(p, \rho) \neq \emptyset$ and $E \subseteq \bigcup_{p \in \mathcal{S}} \mathcal{C}(p, \rho)$

An example of covering discretization is the supercover discretization $\Delta_{\mathcal{S C}}$ which associates to every $X \in \mathcal{P}\left(\mathbb{R}^{n}\right)$ the set of all $p \in \mathcal{D}_{\rho}$ such that $\mathcal{C}(p, \rho)$ intersects $X$ (see Figure 2):

$$
\forall X \subseteq \mathbb{R}^{n}, \quad \Delta_{\mathcal{S C}}(X, \rho)=\left\{p \in \mathcal{D}_{\rho} \mid C(p, \rho) \cap X \neq \emptyset\right\} .
$$

a)

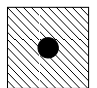

b)

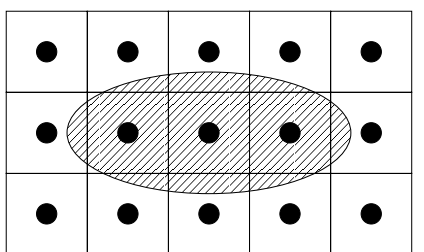

c)

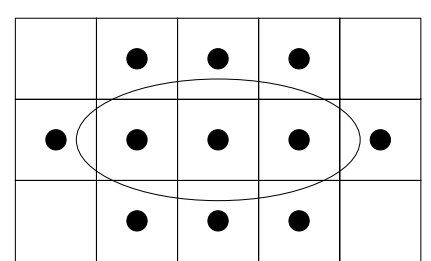

Fig. 2. (a) The cell $C(o, 1)$. (b) A Euclidean set $X$ overlayed with the discrete points $p$ and their cells $C(p, 1)$. (c) $\Delta_{\mathcal{S C}}(X, 1)$

Theorem 3. Let $d$ be an homogeneous metric and let $F \in \mathcal{F}^{\prime}\left(\mathbb{R}^{n}\right)$. If $S$ is a covering discretization of $F$ in $\mathcal{D}_{\rho}$, then $S \in \mathcal{M}_{H}(F, \rho)$.

Definition 11. A metric $d$ on $\mathbb{R}^{2}$ is called strictly homogeneous if $d$ is homogeneous and $\mathcal{B}_{r_{c}(1)}^{d}(0,0) \cap \mathcal{B}_{r_{c}(1)}^{d}(1,1)=\left\{\left(\frac{1}{2}, \frac{1}{2}\right)\right\}$.

In other words, the balls of covering radius centered about diagonally adjacent discrete points intersect only at their corners. For example $d_{p}$ is strictly homogeneous for all $p>1$ and for $p=\infty$.

\section{Algebraic Sets and Diophantine Sets}

In this section, we give the definitions of real algebraic sets, real semi-algebraic sets and diophantine sets. We recall also the decidability of first order logic for elementary algebra corresponding to Tarski's elimination algorithm.

Definition 12. A subset $\mathcal{A}$ of $\mathbb{R}^{n}$ is an algebraic set, if there exists a polynomial $P \in \mathbb{R}\left[X_{1}, \ldots, X_{n}\right]$ such that $\mathcal{A}$ is the set of zeros of $P$ in $\mathbb{R}^{n}$ (i.e. $\mathcal{A}=\{x \in$ $\left.\left.\mathbb{R}^{n} \mid P(x)=0\right\}\right)$.

If the coefficients of $P$ are integers (i.e. $P \in \mathbb{Z}\left[X_{1}, \ldots, X_{n}\right]$ ) then $\mathcal{A}$ is called a $\mathbb{Z}$-algebraic set. 
Definition 13. A subset $\mathcal{S}$ of $\mathbb{R}^{n}$ is a semi-algebraic set, if it can be written as a finite union of set of the form:

$$
\left\{x \in \mathbb{R}^{n} \mid P_{1}(x)=\ldots=P_{l}(x)=0, Q_{1}(x)>0, \ldots, Q_{m}(x)>0\right\},
$$

where $P_{1}, \ldots, P_{l}, Q_{1}, \ldots, Q_{m}$ are in $\mathbb{R}\left[X_{1}, \ldots, X_{n}\right]$. If the coefficients of polynomials involved in a definition of the semi-algebraic set $\mathcal{S}$ are integers (i.e. $P_{i}, Q_{i} \in$ $\left.\mathbb{Z}\left[X_{1}, \ldots, X_{n}\right]\right) \mathcal{S}$ is called a $\mathbb{Z}$-semi-algebraic set.

Actually every subset of $\mathbb{R}^{n}$ defined by a boolean combination (obtained by disjunction, conjunction and negation) of atomic formulas of the form $P(x)>0$, $P(x)<0$ or $P(x)=0$, where $P \in \mathbb{R}\left[X_{1}, \ldots, X_{n}\right]$, is a semi-algebraic set of $\mathbb{R}^{n}$.

Definition 14. A subset $D$ of $\mathbb{Z}^{n}$ is a diophantine set, if there exists a polynomial $P \in \mathbb{Z}\left[X_{1}, \ldots, X_{n}\right]$ such that $D$ is the set of zeros of $P$ in $\mathbb{Z}^{n}$ (i.e. $\left.D=\left\{x \in \mathbb{Z}^{n} \mid P(x)=0\right\}\right)$.

\section{Remarks:}

- A finite intersection of algebraic (resp. diophantine ) sets is an algebraic (resp. diophantine) set. Actually if $A_{1}, \ldots, A_{k}$ are in $\mathbb{R}$ (resp. in $\mathbb{Z}$ ) then $A_{1}=0$ and $A_{2}=0$ and $\ldots$ and $A_{k}=0$ if and only if $A_{1}^{2}+A_{2}^{2}+\ldots+A_{k}^{2}=0$.

- A finite union of algebraic (resp. diophantine ) sets is an algebraic (resp. diophantine) set. Actually if $A_{1}, \ldots, A_{k}$ are in $\mathbb{R}$ (resp. in $\mathbb{Z}$ ) then $A_{1}=0$ or $A_{2}=0$ or $\ldots$ or $A_{k}=0$ if and only if $A_{1} . A_{2} \ldots A_{k}=0$.

In the following theorem, we present the result corresonding to Tarski's elimination algorithm which proves the decidability of first order logic corresponding to elementary algebra.

Theorem 4. [2,14,19,20]

Let $P_{1}\left(X_{1}, \ldots, X_{n}, Y\right), \ldots, P_{k}\left(X_{1}, \ldots, X_{n}, Y\right)$ be a sequence of polynomials in $n+$ 1 variables with coefficients in $\mathbb{Z}$. Then there exists an effectively computable boolean combination $\mathcal{B}\left(X_{1}, \ldots, X_{n}\right)$ of polynomial equations and inequalities in the variables $X_{1}, \ldots, X_{n}$ with coefficients in $\mathbb{Z}$, such that for every $\left(x_{1}, \ldots, x_{n}\right) \in \mathbb{R}^{n}$, the system of polynomial equations and inequalities

$$
\left\{\begin{array}{l}
P_{1}\left(x_{1}, \ldots, x_{n}, Y\right) \succ_{1} 0 \\
\ldots \\
P_{k}\left(x_{1}, \ldots, x_{n}, Y\right) \succ_{k} 0
\end{array}\right.
$$

where $\succ_{i} \in\{=, \geq,>\}$ for $i=1, \ldots, k$,

has a solution $y$ in $\mathbb{R}$ if and only if $\mathcal{B}\left(x_{1}, \ldots, x_{n}\right)$ holds true in $\mathbb{R}$.

Corollary 1. - The boolean combination $\mathcal{B}$ in the last theorem define a semialgebraic set (i.e. $\left\{x \in \mathbb{R}^{n} \mid \mathcal{B}(x)\right.$ holds true in $\left.\mathbb{R}\right\}$ is a semi-algebraic set of $\mathbb{R}^{n}$ ). 
- Let $F$ be a field which is a finite extention of $\mathbb{Q}$ and let $\mathcal{B}^{\prime}\left(X_{1}, \ldots, X_{n}, Y\right)$ be a boolean combination of polynomial equations and inequalities in the variables $X_{1}, \ldots, X_{n}, Y$ with coefficients in $F$; then there exists an effectively computable boolean combination $\mathcal{B}^{\prime \prime}\left(X_{1}, \ldots, X_{n}\right)$ of polynomial equations and inequalities in the variables $X_{1}, \ldots, X_{n}$ with coefficients in $F$, such that for every $\left(x_{1}, \ldots, x_{n}\right) \in \mathbb{R}^{n}$, there exists $y \in \mathbb{R}$ such that $\mathcal{B}^{\prime}\left(x_{1}, \ldots, x_{n}, y\right)$ holds true in $\mathbb{R}$ if and only if $\mathcal{B}^{\prime \prime}\left(x_{1}, \ldots, x_{n}\right)$ holds true in $\mathbb{R} . \mathcal{B}^{\prime \prime}$ is obtained from $\mathcal{B}^{\prime}$ by using Tarski's elimination algorithm corresponding to transformation rules permitting the elimination of the variable $Y$.

\section{Hausdorff Discretizations of Algebraic Sets}

In this section we study the Hausdorff discretizations of real algebraic sets. We prove that some Hausdorff discretizations of real algebraic sets are diophantine sets and we give some decidable and undecidable properties concerning Hausdorff discretizations of real $\mathbb{Z}$-algebraic sets.

Definition 15. A metric $d$ is called algebraic (resp. $\mathbb{Z}$-algebraic) if the unit ball of $d$ is a semi-algebraic (resp. $\mathbb{Z}$-semi-algebraic) set.

For example $d_{p}$ is $\mathbb{Z}$-algebraic for all $p \in \mathbb{Q}$ such that $p \geq 1$ and for $p=\infty$.

The following property is a consequence of the undecidability of diophantine equations ( Hilbert's Tenth Problem ) [10].

Property 1. Let $n \geq 3$. The following problem is undecidable:

Is $r_{H}(\mathcal{A}, \rho)=r_{c}(\rho)$ for a given $\mathbb{Z}$-algebraic set $\mathcal{A}$ of $\mathbb{R}^{n}$, a $\mathbb{Z}$-algebraic metric $d$ on $\mathbb{R}^{n}$ and $\rho \in \mathbb{Q}^{+}$?

The following property is obtained by successive applications of Tarski's elimination algorithm, to a polynomially constrained formula $F_{n}$ whith $n$ unknows corresponding to the problem, until obtaining a formula $F_{0}$ without unknowns.

Property 2. The following problem is decidable:

Is $p \in \Delta_{r}^{d}(\mathcal{A}, \rho)$ for a given $p \in \rho \mathbb{Z}^{n}$, a $\mathbb{Z}$-algebraic set $\mathcal{A}$ of $\mathbb{R}^{n}$, a $\mathbb{Z}$-algebraic metric $d$ on $\mathbb{R}^{n}$ and $r, \rho \in \mathbb{Q}^{+}$?

Theorem 5. Let $\mathcal{A}$ be a $\mathbb{Z}$-algebraic set of $\mathbb{R}^{n}$ and $d$ be a $\mathbb{Z}$-algebraic metric on $\mathbb{R}^{n}$, then

- $\frac{1}{\rho} \Delta_{r}^{d}(\mathcal{A}, \rho)$ is a diophantine set for all $r, \rho \in \mathbb{Q}^{+}$.

- $\frac{1}{\rho} \operatorname{Sk}(\mathcal{A}, \rho)$ is a diophantine set for all $\rho \in \mathbb{Q}^{+}$.

Corollary 2. Let $\mathcal{A}$ be a $\mathbb{Z}$-algebraic set of $\mathbb{R}^{n}$ and $d$ be $\mathbb{Z}$-algebraic metric on $\mathbb{R}^{n}$, then

- The maximal Hausdorff discretization $\Delta_{H}(\mathcal{A}, 1)$ of $\mathcal{A}$ is a diophantine set and $\frac{1}{\rho} \Delta_{H}(\mathcal{A}, \rho)$ is a diophantine set for all $\rho \in \mathbb{Q}^{+}$.

- The supercover discretization $\Delta_{\mathcal{S C}}(\mathcal{A}, 1)$ of $\mathcal{A}$ is a diophantine set and $\frac{1}{\rho} \Delta_{\mathcal{S C}}(\mathcal{A}, \rho)$ is a diophantine set for all $\rho \in \mathbb{Q}^{+}$. 


\section{Hausdorff Discretization of Algebraic Curves}

In this section we study the Hausdorff discretizations of $\mathbb{Z}$-algebraic curves (i.e. a $\mathbb{Z}$-algebraic set of $\mathbb{R}^{2}$ ). We refine this study for straight lines by using Fourier's elimination algorithm because some of the constraits corresponding to this problem are linear. So Fourier's elimination algorithm is a special case of Tarski's elimination algorithm. Our result concerning Hausdorff discretizations of straight lines generalize the results [1] concerning the supercover discretization of straight lines. Several properties of Hausdorff discretizations of straight lines are decidable because these discretizations are defined by linear constraints, which is not the case for Hausdorff discretizations of non linear algebraic sets of $\mathbb{R}^{n}$ for $n \geq 3$ because, it corresponds to diophantine equations on $n$ unknows [10]. Notice that the decidability of a diophantine equation in two unknowns is still an open problem.

Theorem 6. Let $\rho \in \mathbb{R}^{+}$and let $\mathcal{C}$ be a $\mathbb{Z}$-algebraic curve such that the radius of curvature of $\mathcal{C}$ is strictly greater than $\rho$ and let $d$ be an homogeneous and $\mathbb{Z}$-algebraic metric, then

- If $r_{H}(\mathcal{C}, \rho)<r_{c}(\rho)$, then there exists two diophantine sets $D_{1}$ and $D_{2}$ such that

$$
M \in \mathcal{M}_{H}(\mathcal{C}, \rho) \text { if and only if } M=\rho\left(D_{1} \cup S\right) \text { with } S \subseteq D_{2} .
$$

- If $r_{H}(\mathcal{C}, \rho)=r_{c}(\rho)$ then there exists tree diophantine sets $D_{0}, D_{1}$ and $D_{2}$ such that

$M \in \mathcal{M}_{H}(\mathcal{C}, \rho)$ if and only if $M=\rho\left(D_{0} \cup S_{1} \cup S_{2}\right)$ with $S_{1} \subseteq D_{1}, S_{2} \subseteq D_{2}$ and if $p \in\left(D_{1} \backslash S_{1}\right)$, then there exists $S \subseteq \mathcal{V}_{4}(p), S \subseteq S_{2}, \mathcal{B}_{r_{c}(\rho)}^{d}(q) \cap \mathcal{C} \neq \emptyset$ for all $q \in S$ and $\mathcal{C} \cap \mathcal{C}(p, \rho) \subseteq \bigcup_{q \in S} \mathcal{B}_{r_{c}(\rho)}^{d}(q)$, where $\mathcal{V}_{4}(p)$ is the 4-neighbourhood of $p$ in $\rho \mathbb{Z}^{2}$.

Corollary 3. Let $\rho \in \mathbb{R}^{+}$and let $\mathcal{C}$ be a $\mathbb{Z}$-algebraic curve such that the radius of curvature of $\mathcal{C}$ is a strictry greater than $\rho$ and assume that $d$ is strictly homogeneous and $\mathbb{Z}$-algebraic metric, then there exists two diophantine sets $D_{1}$ and $D_{2}$ such that,

$$
M \in \mathcal{M}_{H}(\mathcal{C}, \rho) \text { if and only if } M=\rho\left(D_{1} \cup S\right) \text { with } S \subseteq D_{2} .
$$

\subsection{Hausdorff Discretizations of Straight Lines}

In this subsection we study the Hausdorff discretizations of straight lines for homogeneous metrics. Some of the results of this subsection are proved by using Fourier's elimination algorithm $[4,8,11]$ which transforms linear constraints. Fourier's elimination algorithmis is a special case of Tarski's elimination algorithm. The time complexity of Tarski's elimination algorithm, is in the general case, very high [5]. 
Before giving the results corresponding to Hausdorff discretizations of straight lines, we treat two special cases of horizontal and vertical straight lines.

Let $L$ be a straight line such that $L=\left\{(x, y) \in \mathbb{R}^{2} \mid x+\rho\left(m+\frac{1}{2}\right)=0\right\}$ (resp. $\left.L=\left\{(x, y) \in \mathbb{R}^{2} \mid y+\rho\left(m+\frac{1}{2}\right)=0\right\}\right)$ for $m \in \mathbb{Z}$, then $r_{H}(L, \rho)=r_{c}(\rho)$ and $\forall M \in \mathcal{M}_{H_{d}}(F, \rho) \forall i \in \rho \mathbb{Z}$, if $(\rho m, i) \notin M$ then $(\rho(m+1), i) \in M$ (resp. $\forall i \in \rho \mathbb{Z}, \quad$ if $(i, \rho m) \notin M$ then $(i, \rho(m+1)) \in M)$.

In all the following, we assume that the considered straight lines are not of the last forms.

Definition 16. $S \subset \mathbb{Z}^{2}$ is a periodic set if there exists $p \in \mathbb{Z}^{2}$ and $p \neq(0,0)$ such that $q+p \in S$ for all $q \in S$.

Theorem 7. Let $\mathcal{L}=\left\{(x, y) \in \mathbb{R}^{2} \mid a x+b y+c=0\right\}$ such that $a, b, c \in \rho \mathbb{Z}$

1- If $d$ is an homogeneous metric (the unit ball of $d$ is not necessarily a semialgebraic set) and $r_{H}(\mathcal{L}, \rho)<r_{c}(\rho)$, then there exist two periodic diophantine sets $D_{1}$ and $D_{2}$ such that

$$
M \in \mathcal{M}_{H}(\mathcal{L}, \rho) \text { if and only if } M=\rho\left(D_{1} \cup S\right) \text { with } S \subseteq D_{2} .
$$

2- If $d$ is a strictly homogeneous metric (the unit ball of $d$ is not necessarily a semi-algebraic set), then there exist two periodic diophantine sets $D_{1}$ and $D_{2}$ such that

$$
M \in \mathcal{M}_{H}(\mathcal{L}, \rho) \text { if and only if } M=\rho\left(D_{1} \cup S\right) \text { with } S \subseteq D_{2} .
$$

\section{Sketch of proof:}

This theorem is a consequence of the following two facts:

$\mathcal{L}=\left\{(x, y) \in \mathbb{R}^{2} \mid a x+b y+c=0\right\}$,

- Let $r>0$ and $D_{r}(\mathcal{L}, \rho)=\left\{(m, n) \in \rho \mathbb{Z}^{2} \mid \mathcal{L} \cap \operatorname{int}(\mathcal{C}((m, n), r)) \neq \emptyset\right\}$. So, $D_{r}(\mathcal{L}, \rho)$ is the set of $(m, n) \in \rho \mathbb{Z}^{2}$ such that there exists $t, t^{\prime} \in \mathbb{R}$ and

$$
\left\{\begin{array}{l}
a(m+t)+a\left(n+t^{\prime}\right)+c=0 \\
t>-\frac{r}{2} \\
-t>-\frac{r}{2} \\
t^{\prime}>-\frac{r}{2} \\
-t^{\prime}>-\frac{r}{2}
\end{array}\right.
$$

So, $D_{r}(\mathcal{L}, \rho)=\left\{(m, n) \in \rho \mathbb{Z}^{2} \mid-\frac{r}{2}(|a|+|b|)<a m+b n+c<\frac{r}{2}(|a|+|b|)\right\}$. Thus, $\frac{1}{\rho} D_{r}(\mathcal{L}, \rho)$ is a diophantine set and if $(m, n) \in D_{r}(\mathcal{L}, \rho)$ then $(m, n)+z(b,-a) \in$ $D_{r}(\mathcal{L}, \rho)$ for all $z \in \mathbb{Z}$.

- Let $r \geq 0$ and $(m, n) \in \rho \mathbb{Z}^{2}$ such that $\mathcal{B}_{r}^{d}(m, n) \cap \mathcal{L} \neq \emptyset$, then $\mathcal{B}_{r}^{d}((m, n)+$ $z(b,-a)) \cap \mathcal{L} \neq \emptyset$ for all $z \in \mathbb{Z}$. Moreover, if for some $r^{\prime}, \mathcal{L} \cap \operatorname{int}\left(\mathcal{C}\left((m, n), r^{\prime}\right)\right)=\emptyset$, then $\mathcal{L} \cap \operatorname{int}\left(\mathcal{C}\left((m, n)+z(b,-a), r^{\prime}\right)\right)=\emptyset$.

In Figure 3, we illustrate the sets $D_{1}$ and $D_{2}$ of the last theorem. 


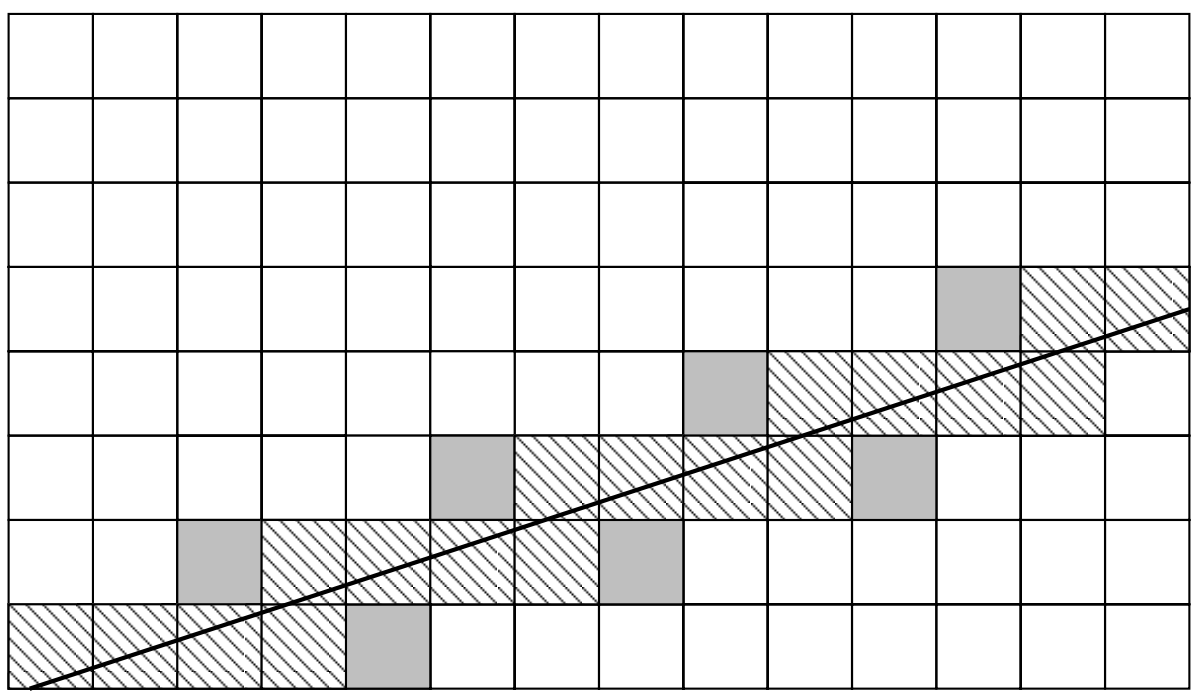

Fig. 3. The hatched squares correspond to points of $D_{1}$ and the grey squares correspond to points of $D_{2}$

\section{Conclusion}

Throughout several papers we have introduced a new framework for the discretization of a non-empty closed set, based on the Hausdorff distance. In this paper we have studed Hausdorff discretizations of real algebraic sets. Actually we have given some decidable and undecidable properties concerning Hausdorff discretizations of algebraic sets and we have proved that some Hausdorff discretizations of algebraic sets are diophantine sets. We have refined the last results for algebraic curves and more precisely for straight lines. Some of these results are proven by using the Tarski's elimination algorithm concerning the decidability of first order logic for elementary algebra.

We intend to do further investigations on:

- effective computation of Hausdorff discretizations of non linear algebraic sets;

- topological properties of Hausdorff discretizations of algebraic sets;

- complexity of representation of some Hausdorff discretizations of algebraic sets.

\section{References}

1. E. Andres. Standard Cover: a new class of discrete primitives. Internal Report, IRCOM, Université de Poitiers. 100, 107

2. J. Bochnak, M. Coste, M.-F. Roy. Real Algebraic Geometry. Serie of Modern Surveys in Mathematics, Vol. 36, Springer, 1998. 105 
3. H. Busemann The geometry of geodesics. Academec Press, New York, 1955. 100, 101

4. J. B. J. Fourier. Solution d'une question particulière du calcul des inégalités. Oeuvre II, Paris, pp. 317-328, 1826. 107

5. D. Grigor'ev, N. Vorobjov. Solving systems of polynomial inequalities in subexponential time. J. Symbolic Comput., Vol. 5, pp. 37-64, 1988. 100, 107

6. F. Haudorff. Set Theory. Chelsea, New York, 1962. 100, 101

7. J. G. Hocking and G. S. Young Topology. Dover Publications Inc., New York, 1988. 100

8. H. W. Kuhn. Solvability and consistency for linear equations and inequalities. Amer. Math. Monthly, Vol. 63, pp 217-232, 1956. 107

9. L. Hörmander. The analysis of linear partial differential operators. Springer-Verlag Berlin, Vol. 2, 1983. 99

10. Y. Matiiassevitch. Enumerable sets are diophantine. Doklady Akad.Nauk SSSR, Vol. 191, pp 279-282, 1970 (English translation : Soviet Math. Doklady, pp 354357, 1970). 106, 107

11. T. S. Motzkin. Beitrage zur theorie der linearen ungleichungen. Azriel : Jerusalem, 1936. 107

12. C. Ronse and M. Tajine. Discretization in Hausdorff Space. Journal of Mathematical Imaging \& Vision, Vol. 12, no 3, pp. 219-242, 2000. 99, 100, 101

13. C. Ronse and M. Tajine. Hausdorff discretization for cellular distances, and its relation to cover and supercover discretization. To be revised, 2000. 99, 101

14. A. Seidenberg. A new decision method for elementary algebra. Ann. of Math., Vol. 60, pp 365-374, 1954. 99, 105

15. M. Tajine and C. Ronse. Preservation of topology by Hausdorff discretization and comparison to other discretization schemes. Submitted, 1999. 99, 100, 101, 103

16. M. Tajine and C. Ronse. Hausdorff sampling of closed sets in a boundedly compact space. In preparation, 2000. 99

17. M. Tajine and C. Ronse. Topological Properties of Hausdorff discretizations. International Symposium on Mathematical Morphology 2000 (ISMM'2000), Palo Alto CA, USA. Kluwer Academic Publishers, pp. 41-50, 2000. 99

18. M. Tajine, D. Wagner and C. Ronse. Hausdorff discretization and its comparison with other discretization schemes. DGCI'99, Paris, LNCS Springer-Verlag, Vol. 1568, pp. 399-410, 1999. 99

19. A. Tarski. Sur les ensembles définissables de nombres réels. Fund. Math., Vol. 17, pp. 210-239, 1931. 99, 105

20. A. Tarski. A decision method for elementary algebra and geometry. Tech. Rep., University of California Press, Berkeley and Los Angeles, 1951. 99, 105

21. D. Wagner. Distance de Hausdorff et problème discret-continu. Mémoire de D. E. A. (M.Sc. Dissertation), Université Louis Pasteur, Strasbourg (France). 1997. 99, 100,101

22. D. Wagner, M. Tajine and C. Ronse. An approach to discretization based on the Hausdorff metric.ISMM'1998. Kluwer Academic Publishers. pp. 67-74, 1998. 99, 100, 101 